\title{
ON ABSORBING BOUNDARY CONDITIONS FOR LINEARIZED EULER EQUATIONS BY A PERFECTLY MATCHED LAYER
}

\author{
FANG Q. Hu \\ Department of Mathematics and Statistics, Old Dominion University \\ Norfolk, VA 23529
}

\begin{abstract}
Recently, Berenger introduced a Perfectly Matched Layer (PML) technique for absorbing electromagnetic waves. In the present paper, a perfectly matched layer is proposed for absorbing out-going two-dimensional waves in a uniform mean flow, governed by linearized Euler equations. It is well known that the linearized Euler equations support acoustic waves, which travel with the speed of sound relative to the mean flow, and vorticity and entropy waves, which travel with the mean flow. The PML equations to be used at a region adjacent to the artificial boundary for absorbing these linear waves are defined. Plane wave solutions to the PML equations are developed and wave propagation and absorption properties are given. It is shown that the theoretical reflection coefficients at an interface between the Euler and PML domains are zero, independent of the angle of incidence and frequency of the waves. As such, the present study points out a possible alternative approach for absorbing out-going waves of the Euler equations with little or no reflection in computation. Numerical examples that demonstrate the validity of the proposed PML equations are also presented.
\end{abstract}

This work was supported by the National Aeronautics and Space Administration under NASA contract NAS1-19480 while the author was in residence at the Institute for Computer Applications in Science and Engineering, NASA Langley Research Center, Hampton, VA 23681, USA. This work was also supported by a summer research fellowship of Old Dominion University, Norfolk, VA 23529. 


\section{INTRODUCTION}

Numerical boundary conditions have always been an important issue in computational fluid dynamics. In problems involving wave radiation and convection, such as in computational acoustics, the physical domains are often necessarily truncated due to the limitation of a finite computational domain. Thus, at these artificial boundaries, numerical non-reflecting or absorbing boundary conditions are needed so that the out-going waves are not reflected. Various computational techniques have been developed in the past to minimize the reflection of out-going waves. They include the out-flow boundary conditions based on the characteristics of the Euler equations $[1,2]$ and radiation boundary conditions based on the far field asymptotic solutions $[3,4,5,6]$. A recent review can be found in reference [7]. In addition, a buffer zone technique has been developed in which the mean flow is altered gradually to be supersonic in a buffer region adjacent to the artificial boundary [8, $9]$.

Recently, Berenger introduced a Perfectly Matched Layer (PML) technique for absorbing electromagnetic waves in a finite difference method of solving the Maxwell equations [10]. In this approach, a PML medium of certain depth is introduced in a region adjacent to the artificial boundary of a computational domain. The equations for the PML medium are designed such that the out-going electromagnetic waves are absorbed by the layer with no reflection (theoretically). The novelty of the technique lies in the way the PML equations are constructed. Numerical studies have shown that the PML technique dramatically reduces the reflection of out-going waves when compared to several previous methods in electromagnetics [10,11].

In this paper, a perfectly matched layer is proposed for absorbing out-going waves of the linearized Euler equations for two-dimensional problems. It is well known that the Euler equations linearized around a uniform mean flow support acoustic waves as well as vorticity and entropy waves. The acoustic waves travel with the speed of sound relative to the mean flow, while the vorticity and entropy waves convect downstream with the mean flow. To apply the PML technique, the computational domain is divided into the interior domain, where the Euler equations are used, and PML domains adjacent to artificial boundaries where the proposed PML equations are to be used (Figure 1). The purpose of the PML domain is to absorb the out-going waves. It will be shown that the theoretical reflection coefficients for incident linear waves at an interface between the interior domain and a PML domain are zero and the amplitudes of waves that enter the PML domain decrease exponentially. It will also be shown that it is true independent of the angle of incidence and frequency of the waves.

In the next section, equations for the PML domains are defined. In section 3, plane wave solutions of the PML equations are developed and wave propagation and absorption properties are given. Then, the theoretical reflection and transmission coefficients at interfaces are calculated in

section 4. Numerical examples that demonstrate the validity of the proposed PML equations are provided in section 5. Section 6 contains the conclusions. 


\section{PERFECTLY MATCHED LAYER EQUATIONS}

We consider the two-dimensional linearized Euler equations with a uniform mean flow :

$$
\begin{aligned}
& \frac{\partial u}{\partial t}+M \frac{\partial u}{\partial x}=-\frac{\partial p}{\partial x} \\
& \frac{\partial v}{\partial t}+M \frac{\partial v}{\partial x}=-\frac{\partial p}{\partial y} \\
& \frac{\partial p}{\partial t}+M \frac{\partial p}{\partial x}=-\left(\frac{\partial u}{\partial x}+\frac{\partial v}{\partial y}\right) \\
& \frac{\partial \rho}{\partial t}+M \frac{\partial \rho}{\partial x}=-\left(\frac{\partial u}{\partial x}+\frac{\partial v}{\partial y}\right)
\end{aligned}
$$

in which $u$ and $v$ are the velocity components in the $x$ and $y$ directions respectively, $p$ is the pressure, and $\rho$ is the density. The velocities have been non-dimensionalized by the speed of sound $a$, the density by $\bar{\rho}$ and the pressure by $\bar{\rho} a^{2}$, where $\bar{\rho}$ is the mean density. For simplicity, a mean flow of Mach number $M$ in the direction of the $x$-axis has been assumed. Situations of a mean flow not aligned with the $x$-axis are discussed in the Appendix. It is also assumed that the mean flow is subsonic, i.e., $M<1$.

Equations (1.1)-(1.4) support acoustic waves, which travel with the speed of sound relative to the mean flow, and vorticity and entropy waves, which travel with the mean flow. Our aim is to define a perfectly matched layer to be used at a region adjacent to the artificial boundary which absorbs the out-going acoustic, vorticity and entropy waves with little or no reflection.

Different from [10], here we split $u, v, p$ and $\rho$ in equations (1.1)-(1.4) into sub-components $u_{1}, u_{2}, v_{1}, v_{2}, p_{1}, p_{2}$ and $\rho_{1}, \rho_{2}$. We define the following equations for the perfectly matched layer (PML) :

$$
\begin{aligned}
& \frac{\partial u_{1}}{\partial t}+\sigma_{x} u_{1}=-\frac{\partial\left(p_{1}+p_{2}\right)}{\partial x} \\
& \frac{\partial u_{2}}{\partial t}+\sigma_{x} u_{2}=-M \frac{\partial\left(u_{1}+u_{2}\right)}{\partial x} \\
& \frac{\partial v_{1}}{\partial t}+\sigma_{y} v_{1}=-\frac{\partial\left(p_{1}+p_{2}\right)}{\partial y} \\
& \frac{\partial v_{2}}{\partial t}+\sigma_{x} v_{2}=-M \frac{\partial\left(v_{1}+v_{2}\right)}{\partial x} \\
& \frac{\partial p_{1}}{\partial t}+\sigma_{x} p_{1}=-\frac{\partial\left(u_{1}+u_{2}\right)}{\partial x}-M \frac{\partial\left(p_{1}+p_{2}\right)}{\partial x} \\
& \frac{\partial p_{2}}{\partial t}+\sigma_{y} p_{2}=-\frac{\partial\left(v_{1}+v_{2}\right)}{\partial y} \\
& \frac{\partial \rho_{1}}{\partial t}+\sigma_{x} \rho_{1}=-\frac{\partial\left(u_{1}+u_{2}\right)}{\partial x}-M \frac{\partial\left(\rho_{1}+\rho_{2}\right)}{\partial x} \\
& \frac{\partial \rho_{2}}{\partial t}+\sigma_{y} \rho_{2}=-\frac{\partial\left(v_{1}+v_{2}\right)}{\partial y}
\end{aligned}
$$

In the above, $\sigma_{x}$ and $\sigma_{y}$ have been introduced for the absorption of waves in the layer. They will be called absorption coefficients in this paper and are assumed to be greater than or equal 
to zero. It is to be noted that, when $\sigma_{x}=\sigma_{y}=0$, equations (2.1)-(2.8) can be reduced to the Euler equations (1.1)-(1.4) with $u=u_{1}+u_{2}, v=v_{1}+v_{2}, p=p_{1}+p_{2}$ and $\rho=\rho_{1}+\rho_{2}$. Thus the Euler equations are a special case of the PML equations. Moreover, the spatial derivatives involve only the total $u, v, p$ and $\rho$ which are assumed to be continuous at interfaces. We also note that two kinds of interfaces are created, namely, the interfaces between the interior domain and a PML domain and those between two PML domains, as shown in Figure 2. The former, of course, can be regarded as a special case of the later. In the next two sections, we show the wave propagation and absorption properties within a perfectly matched layer defined above and calculate the reflection and transmission coefficients at an interface between two PML domains.

\section{Plane WAVES in a PERFECTLy MATCHED LAYER}

Let a plane wave in the PML domain be expressed as

$$
\left[u_{1}, u_{2}, v_{1}, v_{2}, p_{1}, p_{2}, \rho_{1}, \rho_{2}\right]=\left[u_{10}, u_{20}, v_{10}, v_{20}, p_{10}, p_{20}, \rho_{10}, \rho_{20}\right] e^{i\left(k_{x} x+k_{y} y-\omega t\right)}
$$

in which a subscript 0 has been used to denote the amplitudes of the components. By substituting (3) into (2.1)-(2.8), we get

$$
\begin{aligned}
& \left(\omega+i \sigma_{x}\right) u_{10}=k_{x}\left(p_{10}+p_{20}\right) \\
& \left(\omega+i \sigma_{x}\right) u_{20}=k_{x} M\left(u_{10}+u_{20}\right) \\
& \left(\omega+i \sigma_{y}\right) v_{10}=k_{y}\left(p_{10}+p_{20}\right) \\
& \left(\omega+i \sigma_{x}\right) v_{20}=k_{x} M\left(v_{10}+v_{20}\right) \\
& \left(\omega+i \sigma_{x}\right) p_{10}=k_{x}\left(u_{10}+u_{20}\right)+k_{x} M\left(p_{10}+p_{20}\right) \\
& \left(\omega+i \sigma_{y}\right) p_{20}=k_{y}\left(v_{10}+v_{20}\right) \\
& \left(\omega+i \sigma_{x}\right) \rho_{10}=k_{x}\left(u_{10}+u_{20}\right)+k_{x} M\left(\rho_{10}+\rho_{20}\right) \\
& \left(\omega+i \sigma_{y}\right) \rho_{20}=k_{y}\left(v_{10}+v_{20}\right)
\end{aligned}
$$

It will be shown below that equations (4.1)-(4.8) support acoustic waves, when $\omega-k_{x} M+i \sigma_{x} \neq$ 0 , and vorticity and entropy waves, when $\omega-k_{x} M+i \sigma_{x}=0$.

\subsection{Acoustic Waves}

When $\omega-k_{x} M+i \sigma_{x} \neq 0$, it is easy to find that the amplitudes of the components in (3) can be expressed in terms of $u_{10}$ and $v_{10}$ as follows:

$$
\begin{aligned}
& u_{20}=\frac{k_{x} M}{\omega-k_{x} M+i \sigma_{x}} u_{10} \\
& v_{20}=\frac{k_{x} M}{\omega-k_{x} M+i \sigma_{x}} v_{10}
\end{aligned}
$$




$$
\begin{aligned}
p_{10} & =\frac{1}{\left(\omega-k_{x} M+i \sigma_{x}\right)^{2}}\left(k_{x}\left(\omega+i \sigma_{x}\right) u_{10}+k_{x} k_{y} M \frac{\omega+i \sigma_{x}}{\omega+i \sigma_{y}} v_{10}\right) \\
p_{20} & =\frac{k_{y}}{\omega-k_{x} M+i \sigma_{x}} \frac{\omega+i \sigma_{x}}{\omega+i \sigma_{y}} v_{10} \\
\rho_{10} & =\frac{1}{\left(\omega-k_{x} M+i \sigma_{x}\right)^{2}}\left(k_{x}\left(\omega+i \sigma_{x}\right) u_{10}+k_{x} k_{y} M \frac{\omega+i \sigma_{x}}{\omega+i \sigma_{y}} v_{10}\right) \\
\rho_{20} & =\frac{k_{y}}{\omega-k_{x} M+i \sigma_{x}} \frac{\omega+i \sigma_{x}}{\omega+i \sigma_{y}} v_{10}
\end{aligned}
$$

In addition, from (4.1) and (4.3), we have the following relation :

$$
\frac{\omega+i \sigma_{x}}{\omega+i \sigma_{y}} \frac{u_{10}}{v_{10}}=\frac{k_{x}}{k_{y}}
$$

Now, by substituting (5.3)-(5.4) into (4.1) and (4.3), we get

$$
\begin{aligned}
\left(\omega+i \sigma_{x}\right) u_{10} & =\frac{k_{x}^{2}\left(\omega+i \sigma_{x}\right)\left(\omega+i \sigma_{y}\right) u_{10}+k_{x} k_{y}\left(\omega+i \sigma_{x}\right)^{2} v_{10}}{\left(\omega-k_{x} M+i \sigma_{x}\right)^{2}\left(\omega+i \sigma_{y}\right)}, \\
\left(\omega+i \sigma_{y}\right) v_{10} & =\frac{k_{x} k_{y}\left(\omega+i \sigma_{x}\right)\left(\omega+i \sigma_{y}\right) u_{10}+k_{y}^{2}\left(\omega+i \sigma_{x}\right)^{2} v_{10}}{\left(\omega-k_{x} M+i \sigma_{x}\right)^{2}\left(\omega+i \sigma_{y}\right)},
\end{aligned}
$$

respectively. For (7.1) and (7.2) to have non-trivial solutions for $u_{10}$ and $v_{10}$, it is found that the following dispersion relation for $k_{x}, k_{y}$ and $\omega$ has to hold:

$$
\left(\omega-k_{x} M+i \sigma_{x}\right)^{2}\left(\omega+i \sigma_{y}\right)^{2}-k_{x}^{2}\left(\omega+i \sigma_{y}\right)^{2}-k_{y}^{2}\left(\omega+i \sigma_{x}\right)^{2}=0 .
$$

However, it has been found more convenient to express $k_{x}$ and $k_{y}$ in terms of $u_{10}$ and $v_{10}$. On eliminating $k_{y}$ and $k_{x}$ in the numerators of (7.1) and (7.2), respectively, using equation (6), we obtain

$$
\begin{aligned}
& k_{x}= \pm\left(\omega-k_{x} M+i \sigma_{x}\right) \frac{u_{10}}{\sqrt{u_{10}^{2}+v_{10}^{2}}}, \\
& k_{y}= \pm\left(\omega-k_{x} M \frac{\omega+i \sigma_{y}}{\omega+i \sigma_{x}}+i \sigma_{y}\right) \frac{v_{10}}{\sqrt{u_{10}^{2}+v_{10}^{2}}} .
\end{aligned}
$$

The positive and negative signs indicate the direction of wave propagation. The positive sign will be taken in the discussions followed. For convenience, we express $u_{10}$ and $v_{10}$ as

$$
\begin{aligned}
& u_{10}=A \cos \phi, \\
& v_{10}=A \sin \phi,
\end{aligned}
$$

where $A$ is a complex number and $\phi$ is a real number. This is possible when $u_{10}$ and $v_{10}$ have the same phase, i.e., when $u_{10} / v_{10}$ is real. It will be shown in Appendix A that $u_{10} / v_{10}$ is indeed real 
for solutions of the Euler equations. Thus, it is sufficient to consider only solutions where $u_{10} / v_{10}$ is real.

Substituting (10.1)-(10.2) into (9.1)-(9.2) and solving for $k_{x}$ and $k_{y}$, we get

$$
\begin{aligned}
& k_{x}=\frac{\omega+i \sigma_{x}}{1+M \cos \phi} \cos \phi, \\
& k_{y}=\frac{\omega+i \sigma_{y}}{1+M \cos \phi} \sin \phi .
\end{aligned}
$$

With these expressions, further simplifications can be made for the amplitudes in (5.1)-(5.6). As a result, the plane wave solution to (4.1)-(4.8) can be expressed as

$$
\left(\begin{array}{c}
u_{1} \\
u_{2} \\
v_{1} \\
v_{2} \\
p_{1} \\
p_{2} \\
\rho_{1} \\
\rho_{2}
\end{array}\right)=A\left(\begin{array}{c}
\cos \phi \\
M \cos ^{2} \phi \\
\sin \phi \\
M \cos \phi \sin \phi \\
\cos ^{2} \phi+M \cos \phi \\
\sin ^{2} \phi \\
\cos ^{2} \phi+M \cos \phi \\
\sin ^{2} \phi
\end{array}\right) e^{i \omega\left(\frac{\cos \phi}{1+M \cos \phi} x+\frac{\sin \phi}{1+M \cos \phi} y-t\right)} e^{-\frac{\sigma_{x} \cos \phi}{1+M \cos \phi} x-\frac{\sigma_{y} \sin \phi}{1+M \cos \phi} y}
$$

It is easy to see that the above expression represents a wave propagating with the speed of sound (which is unity in the non-dimensional variables) relative to the mean flow in the direction making an angle $\phi$ with respect to the $x$-axis [12]. This solution, thus, represents the acoustic wave in the PML domain. Furthermore, when $\sigma_{x}$ or $\sigma_{y}$ is not zero, the magnitude of the wave decreases exponentially as it propagates in the $x$ or $y$ direction respectively.

\subsection{Vorticity and Entropy Waves}

When $\omega-k_{x} M+i \sigma_{x}=0$, it follows immediately that

$$
k_{x}=\frac{\omega+i \sigma_{x}}{M} .
$$

Under this condition, it also follows that $u_{10}=0, v_{10}=0$ and $p_{10}+p_{20}=0$ by (4.2), (4.4) and (4.1) or (4.3), respectively. Equations (4.1)-(4.8) now admit vorticity-like and entropy-like solutions. For convenience, we express $u_{20}, v_{20}$ and $\rho_{10}$ as

$$
\begin{aligned}
& u_{20}=-B \sin \psi, \\
& v_{20}=B \cos \psi, \\
& \rho_{10}=C .
\end{aligned}
$$


Then, $k_{y}$ for the vorticity wave is found to be

$$
k_{y}=\frac{\omega+i \sigma_{y}}{M} \tan \psi
$$

and the plane wave solution to (4.1)-(4.8) can be expressed as

$$
\left(\begin{array}{l}
u_{1} \\
u_{2} \\
v_{1} \\
v_{2} \\
p_{1} \\
p_{2} \\
\rho_{1} \\
\rho_{2}
\end{array}\right)=\left(\begin{array}{c}
0 \\
-B \sin \psi \\
0 \\
B \cos \psi \\
-B(\sin \psi) / M \\
B(\sin \psi) / M \\
C \\
B(\sin \psi) / M
\end{array}\right) e^{i \omega\left(\frac{1}{M} x+\frac{\tan \psi}{M} y-t\right)} e^{-\frac{\sigma_{x}}{M} x-\frac{\sigma_{y} \tan \psi}{M} y}
$$

We remark that the constants $B$ and $C$ independently represent the vorticity and the entropy waves. Since each component is of the form $f\left(\frac{x}{M}-t, y\right) e^{-\frac{\sigma_{x}}{M} x}$, the waves are convected with the mean flow at a speed that is equal to $M$. Furthermore, when $\sigma_{x}$ is greater than zero, the magnitude is decreased exponentially in $x$. It is worth pointing out that the vorticity waves do not contribute to the $u_{1}$ and $v_{1}$ components in the PML equations.

\section{REFLECTION AND TRANSMISSION AT AN INTERFACE BETWEEN TWO PERFECTLY MATCHED LAYERS}

We now consider the wave reflection and transmission at an interface between two PML domains. This, of course, includes the interface between the interior domain and a PML domain. As in the PML technique for electromagnetic waves [10], the absorbing coefficients, $\sigma_{x}$ and $\sigma_{y}$, will be chosen such that $\sigma_{y}$ is the same across an interface normal to $x$ and $\sigma_{x}$ is the same normal to $y$. Since the Euler equations for the interior domain can be considered as PML equations with both absorption coefficients being zero, $\sigma_{y}$ or $\sigma_{x}$ will be consequently zero across an interface normal to $x$ or $y$ between an interior domain and a PML domain. This is as shown in Figure 2. In what follows we show that the reflection coefficient at an interface downstream normal to $x$ is zero for incident acoustic, vorticity and entropy waves. Similar results can be established analogously for other interfaces.

Let the interface be located downstream at $x=0$ and the absorption coefficients be $\sigma_{x 1}$ and $\sigma_{y}$ on one side and $\sigma_{x 2}$ and $\sigma_{y}$ on the other (figure 3). For a subsonic mean flow, the possible reflection is an acoustic wave. Then, by the results of previous section, the incident, reflected and transmitted waves can be expressed as follows:

(a) incident wave : 


$$
\begin{aligned}
& \left(\begin{array}{c}
u_{1} \\
u_{2} \\
v_{1} \\
v_{2} \\
p_{1} \\
p_{2} \\
\rho_{1} \\
\rho_{2}
\end{array}\right)=A_{i}\left(\begin{array}{c}
\cos \phi_{i} \\
M \cos ^{2} \phi_{i} \\
\sin \phi_{i} \\
M \cos \phi_{i} \sin \phi_{i} \\
\cos ^{2} \phi_{i}+M \cos \phi_{i} \\
\sin ^{2} \phi_{i} \\
\cos ^{2} \phi_{i}+M \cos \phi_{i} \\
\sin ^{2} \phi_{i}
\end{array}\right) e^{i \omega\left(\frac{\cos \phi_{i}}{1+M \cos \phi_{i}} x+\frac{\sin \phi_{i}}{1+M \cos \phi_{i}} y-t\right)} e^{-\frac{\sigma_{x 1} \cos \phi_{i}}{1+M \cos \phi_{i}} x-\frac{\sigma_{y} \sin \phi_{i}}{1+M \cos \phi_{i}} y} \\
& +\left(\begin{array}{c}
0 \\
-B_{i} \sin \psi_{i} \\
0 \\
B_{i} \cos \psi_{i} \\
-B_{i} \sin \psi_{i} / M \\
B_{i} \sin \psi_{i} / M \\
C \\
B_{i} \sin \psi_{i} / M
\end{array}\right) e^{i \omega\left(\frac{1}{M} x+\frac{\left.\tan \psi_{i} y-t\right)}{M} y-t\right)} e^{-\frac{\sigma_{x 1} x-\frac{\sigma_{y} \tan \psi_{i}}{M} y}{M}}
\end{aligned}
$$

(b) reflected wave:

$$
\left(\begin{array}{c}
u_{1} \\
u_{2} \\
v_{1} \\
v_{2} \\
p_{1} \\
p_{2} \\
\rho_{1} \\
\rho_{2}
\end{array}\right)=A_{r}\left(\begin{array}{c}
-\cos \phi_{r} \\
M \cos ^{2} \phi_{r} \\
\sin \phi_{r} \\
-M \cos \phi_{r} \sin \phi_{r} \\
\cos ^{2} \phi_{r}-M \cos \phi_{r} \\
\sin ^{2} \phi_{r} \\
\cos ^{2} \phi_{r}-M \cos \phi_{r} \\
\sin ^{2} \phi_{r}
\end{array}\right) e^{i \omega\left(-\frac{\cos \phi_{r}}{1-M \cos \phi_{r}} x+\frac{\sin \phi_{r}}{1-M \cos \phi_{r}} y-t\right)} e^{\frac{\sigma_{r 1} \cos \phi_{r}}{1-M \cos \phi_{r}} x-\frac{\sigma_{y} \sin \phi_{r}}{1-M \cos \phi_{r}} y}
$$

(c) transmitted wave:

$$
\begin{aligned}
\left(\begin{array}{c}
u_{1} \\
u_{2} \\
v_{1} \\
v_{2} \\
p_{1} \\
p_{2} \\
\rho_{1} \\
\rho_{2}
\end{array}\right)=A_{t}\left(\begin{array}{c}
\cos \phi_{t} \\
M \cos ^{2} \phi_{t} \\
\sin \phi_{t} \\
M \cos \phi_{t} \sin \phi_{t} \\
\cos ^{2} \phi_{t}+M \cos \phi_{t} \\
\sin ^{2} \phi_{t} \\
\cos ^{2} \phi_{t}+M \cos \phi_{t} \\
\sin ^{2} \phi_{t}
\end{array}\right) e^{i \omega\left(\frac{\cos \phi_{t}}{1+M \cos \phi_{t}} x+\frac{\sin \phi_{t}}{1+M \cos \phi_{t}} y-t\right)} e^{-\frac{\sigma_{r 2} \cos \phi_{t}}{1+M \cos \phi_{t}} x-\frac{\sigma_{y} \sin \phi_{t}}{1+M \cos \phi_{t}} y} \\
+\left(\begin{array}{c}
0 \\
-B_{t} \sin \psi_{t} \\
0 \\
B_{t} \cos \psi_{t} \\
-B_{t} \sin \psi_{t} / M \\
B_{t} \sin \psi_{t} / M \\
C_{t} \\
B_{t} \sin \psi_{t} / M
\end{array}\right) e^{i \omega\left(\frac{1}{M} x+\frac{\tan \psi_{t}}{M} y-t\right)} e^{-\frac{\sigma_{x 2}}{M} x-\frac{\sigma_{y} \tan \psi_{t}}{M} y}
\end{aligned}
$$


The angles of the acoustic waves are as indicated in Figure 3.

At the interface, we impose the condition that $u_{1}+u_{2}, v_{1}+v_{2}, p_{1}+p_{2}$ and $\rho_{1}+\rho_{2}$ be continuous. Since this continuity is true for all values of $y$ along the interface, it follows that the coefficients of $y$ in the exponents of (16.1)-(16.3) must be the same for all the incident, reflected and transmitted waves. This yields

$$
\begin{aligned}
\frac{\sin \phi_{r}}{1-M \cos \phi_{r}} & =\frac{\sin \phi_{i}}{1+M \cos \phi_{i}}, \\
\frac{\sin \phi_{t}}{1+M \cos \phi_{t}} & =\frac{\sin \phi_{i}}{1+M \cos \phi_{i}}, \\
\tan \psi_{t} & =\tan \psi_{i} .
\end{aligned}
$$

(Here, $\tan \psi_{i}=\sin \phi_{i} /\left(1+M \cos \phi_{i}\right)$ has been assumed for the incident waves.) From above, it is found that

$$
\begin{aligned}
\phi_{r} & =2 \tan ^{-1}\left(\frac{1-M}{1+M} \tan \frac{\phi_{i}}{2}\right), \\
\phi_{t} & =\phi_{i}, \\
\psi_{t} & =\psi_{i} .
\end{aligned}
$$

Furthermore, by the continuity of $u_{1}+u_{2}, v_{1}+v_{2}, p_{1}+p_{2}$ and $\rho_{1}+\rho_{2}$, we have

$$
\begin{gathered}
A_{i}\left(1+M \cos \phi_{i}\right) \cos \phi_{i}-A_{r}\left(1-M \cos \phi_{r}\right) \cos \phi_{r}-B_{i} \sin \psi_{i}=A_{t}\left(1+M \cos \phi_{t}\right) \cos \phi_{t}-B_{t} \sin \psi_{t}, \\
A_{i}\left(1+M \cos \phi_{i}\right) \sin \phi_{i}+A_{r}\left(1-M \cos \phi_{r}\right) \sin \phi_{r}+B_{i} \cos \psi_{i}=A_{t}\left(1+M \cos \phi_{t}\right) \sin \phi_{t}+B_{t} \cos \psi_{t}, \\
A_{i}\left(1+M \cos \phi_{i}\right)+A_{r}\left(1-M \cos \phi_{r}\right)=A_{t}\left(1+M \cos \phi_{t}\right),
\end{gathered}
$$

and

$$
A_{i}\left(1+M \cos \phi_{i}\right)+A_{r}\left(1-M \cos \phi_{r}\right)+B_{i} \frac{\sin \psi_{i}}{M}+C_{i}=A_{t}\left(1+M \cos \phi_{t}\right)+B_{t} \frac{\sin \psi_{t}}{M}+C_{t} .
$$

Applying (17.1) and (17.3), the above equations can be rewritten as a linear system of four homogeneous equations for $A_{i}-A_{t}, A_{r}, B_{i}-B_{t}$ and $C_{i}-C_{t}$ and the coefficient determinant is found to be

$$
2\left(1+M \cos \phi_{i}\right)\left(1-M \cos \phi_{r}\right) \cos \left(\frac{\phi_{i}+\phi_{r}}{2}\right) \cos \left(\psi_{i}-\frac{\phi_{i}-\phi_{r}}{2}\right) .
$$

It is straightforward to verify that the above is not zero for any angle of incidence and, thus, the only solution is

$$
\begin{aligned}
& A_{r}=0, \\
& A_{t}=A_{i}, \\
& B_{t}=B_{i}, \\
& C_{t}=C_{i} .
\end{aligned}
$$


Therefore, equations (17.1)-(17.3) and (18.1)-(18.4) demonstrate that at an interface between two PML domains downstream normal to the $x$-axis with absorption coefficients $\left(\sigma_{x 1}, \sigma_{y}\right)$ and $\left(\sigma_{x 2}, \sigma_{y}\right)$ respectively, the reflection is null and the transmitted waves maintain the same direction and amplitude as the incident waves at the interface. This has been shown to be independent of the angle of incidence and frequency of the waves.

\section{NUMERICAL EXAMPLES}

Three numerical examples are presented in which the mean flow is in the direction of the $x$ axis or at a $45^{\circ}$ angle with the $x$-axis and Gaussian shaped acoustic, vorticity and entropy pulses are initiated in the interior domain at $t=0$. The first two examples have four open boundaries, while the third example has a solid wall at one boundary. The purpose of these examples is to demonstrate the validity of the proposed PML equations.

The implementation of the PML equations in a finite difference scheme can be straightforward, since the spatial derivatives involve only the total $u, v, p$ and $\rho$. A central difference scheme has been used for spatial discretization in the examples. In particular, a 4th-order 7-point explicit central difference scheme is adopted [6]. Time integration is carried out by a 4th-order RungeKutta scheme optimized for low-dissipation and low-dispersion errors [13]. Since a wide stencil has been used here, the absorption coefficients are varied gradually in the PML domain. Specifically, the following form has been used for $\sigma_{x}$ and $\sigma_{y}$ in the calculations presented:

$$
\sigma=\sigma_{m}\left(\frac{d}{D}\right)^{\beta}
$$

where $D$ is the thickness of the PML domain and $d$ is the distance from the interface with the interior domain.

At the end of a PML domain, certain boundary conditions, such as a solid wall condition or other radiation boundary condition, can be applied. If this is the case, the wave is reflected and, when it re-enters the interior domain, the total absorption factor can be estimated as

$$
e^{-\frac{2 \sigma_{m} D}{\beta+1} \frac{\cos \phi}{1-M^{2} \cos ^{2} \phi}}
$$

for the acoustic wave in a PML domain normal to $x$ [10]. The absorption factor for the vorticity and entropy waves is estimated as

$$
e^{-\frac{\sigma_{m} D}{M(\beta+1)}}
$$

The parameters of the layer can thus be adjusted for desired absorption.

In the first two examples, however, since the numerical solutions are decaying exponentially toward the edges of the computational domain, due to the presence of four PML domains, a periodic boundary condition is used at the end of the PML domains for computing the spatial derivatives. This eliminates the need of backward differences. Consequently, this will result in the out-going 
wave traveling in the two opposite PML domains and the absorption factor for the acoustic wave is now

$$
e^{-\frac{2 \sigma_{m} D}{\beta+1} \frac{\cos \phi}{1 \pm M \cos \phi}}
$$

where the + and - signs are for the right and left traveling waves respectively. We note that the absorption factor for acoustic waves varies with the wave angle. There will be, for instance, little absorption in a layer normal to $x$ for waves with $\phi$ close to $\pm \pi / 2$. These, however, are absorbed by the layers normal to the $y$-axis [10]. Moreover, since the solution is treated as periodic in $x$ and $y$, spectral filtering with FFT is employed.

The initial conditions are adopted from the Benchmark Problems of Computational Aeroacoustics [14]:

$$
\rho=e^{-(\ln 2) \frac{\left(x-x_{a}\right)^{2}+\left(y-y_{a}\right)^{2}}{9}}+0.1 e^{-(\ln 2) \frac{\left(x-x_{b}\right)^{2}+\left(y-y_{b}\right)^{2}}{16}}, p=e^{-(\ln 2) \frac{\left(x-x_{a}\right)^{2}+\left(y-y_{a}\right)^{2}}{9}},
$$

and

$$
u=0.05\left(y-y_{b}\right) e^{-(\ln 2) \frac{\left(x-x_{b}\right)^{2}+\left(y-y_{b}\right)^{2}}{16}}, v=-0.05\left(x-x_{b}\right) e^{-(\ln 2) \frac{\left(x-x_{b}\right)^{2}+\left(y-y_{b}\right)^{2}}{16}} .
$$

The above equations include an acoustic pulse centered at $\left(x_{a}, y_{a}\right)$ and a vorticity and an entropy pulse both centered at $\left(x_{b}, y_{b}\right)$.

In the first example, the mean flow is in the direction of the $x$-axis with $M=0.5$. The computational domain is $[-60,60] \times[-60,60]$ where $\Delta x=\Delta y=1$ and a PML domain of 10 grid points has been used around the four sides. Thus, the interior domain where the Euler equations are applied is $[-50,50] \times[-50,50]$. The initial center of the acoustic pulse is $\left(x_{a}, y_{a}\right)=(-25,0)$ and that of the vorticity and entropy waves is $\left(x_{b}, y_{b}\right)=(25,0)$. For the present calculations, $\sigma_{m}=\beta=2$ has been taken. The contours of computed $\rho$ and $u$ are shown in Figure 4 and 5 for time $t=10,40,50$ and 70. In Figure 6, contour plots of $\rho$ within the PML domain are shown for the right boundary. Clearly, no visible reflection is seen in the contour plots as the pulses exit the boundaries. Comparisons with the exact solution are shown in Figure 7 for density along the line $y=0$. Good agreement is observed. In the second example, the mean flow is in the direction making a $45^{\circ}$ angle with the $x$-axis and $M_{x}=M_{y}=0.5 \sin (\pi / 4)$. The initial locations of the pulses are $\left(x_{a}, y_{a}\right)=(-15,-15)$ and $\left(x_{b}, y_{b}\right)=(30,30)$. Equations given in Appendix $\mathrm{B}$ are used in the PML domains for this example. Contours of $\rho$ and $u$ are shown in Figure 8 and 9 and comparisons with the exact solution along the line $x=y$ are shown in Figure 10. Again, good agreement is found.

To study the effects of the PML domain thickness on the reflection in computation, numerical solutions are computed with different PML domain thicknesses and compared with a reference solution. The reference solution is obtained by using a larger computational domain so that it is not affected by the boundary condition. In Figure 11, the maximum difference of the pressure between the computed and the reference solutions along the line $x=48$ is shown as a function 
of time. This measures the magnitude of the reflected wave at the out-flow boundary. PML domains with $6,8,10$ and 16 grid points have been used. It is seen that reflection is reduced as the PML domain thickness increases. For reference purpose, results using an asymptotic solution based boundary condition are also shown in Figure 11. We note that the asymptotic solution based boundary condition would perform better if the acoustic pulse was initiated at the center of the interior domain and the numerical boundaries placed farther [14]. Considering that the out-going waves have a magnitude around 0.1 , satisfactory results can be obtain with a PML domain thickness as small as 8 grid points.

The third example simulates the reflection of an acoustic pulse by a solid wall with a mean flow $M=0.5$. The computational domain is $[-60,60] \times[0,110]$ and the solid wall is located at $y=0$, where $v=0$ is applied [13]. A PML domain of 10 grid points has been used around the other three sides. At $t=0$, an acoustic pulse of half width 5 is initiated at $\left(x_{a}, y_{a}\right)=(-25,30)$. Since the solution can not be treated as being periodic in the $y$ direction in this example, backward difference schemes of [15] are used for computing $y$ derivatives near the upper and lower boundaries. Pressure contours are shown in Figure 12. It is seen that, although the pulse width is larger than that in the first two examples, the results are satisfactory when the same PML domain thickness is used. The reflection error is also assessed in Figure 13 in which the maximum pressure difference of the computed and the reference solutions along the line $x=48$ is plotted as a function of time. Again, the numerical reflection is reduced as the PML domain thickness increases. The asymptotic solution based boundary condition, however, gives a larger reflection in this case.

\section{CONCLUSIONS}

A perfectly matched layer is proposed for absorbing the linear waves of the Euler equations. Wave propagation and absorption properties are given for the PML equations. It is shown that a PML domain so defined is capable of absorbing out-going acoustic, vorticity and entropy waves with no reflection (theoretically). Moreover, numerical examples that demonstrate the validity of the proposed PML equations are presented.

We remark that, unlike boundary conditions based on asymptotic solutions, it is not necessary to apply the PML equations at far field. Although it results in solving more equations in the PML domains, the extra work is justified by a reduced size of the necessary computational domain. The PML equations also apply where an asymptotic solution is not available. In addition, the no-reflection and absorption properties are independent of the frequency of the waves. This implies that the required layer thickness may be determined independent of the wavelength of the out-going waves. The PML technique also suggests the possibility of using periodic conditions for computing spatial derivatives in certain cases, such as those in the numerical examples. 


\section{APPENDIX A : LINEAR WAVES OF THE EULER EQUATIONS}

Plane wave solutions of (1.1)-(1.4) can be expressed as follows [12]:

$$
\begin{aligned}
\left(\begin{array}{l}
u \\
v \\
p \\
\rho
\end{array}\right) & =A^{\prime}\left(\begin{array}{c}
\cos \phi \\
\sin \phi \\
1 \\
1
\end{array}\right) e^{i \omega\left(\frac{\cos \phi}{1+M \cos \phi} x+\frac{\sin \phi}{1+M \cos \phi} y-t\right)} \\
& +\left(\begin{array}{c}
-B^{\prime} \sin \psi \\
B^{\prime} \cos \psi \\
0 \\
C^{\prime}
\end{array}\right) e^{i \omega\left(\frac{x}{M}+\frac{\tan \psi}{M} y-t\right)}
\end{aligned}
$$

in which $A^{\prime}, B^{\prime}$ and $C^{\prime}$ represent the magnitudes of the acoustic, vorticity and entropy waves, respectively.

It is, of course, possible to rewrite the above as a solution to the PML equations with $\sigma_{x}=\sigma_{y}=$ 0 . The process is straightforward using (4.1)-(4.8) and (5.1)-(5.6). In particular, we note that the vorticity and entropy waves do not contribute to the $u_{10}$ and $v_{10}$ components. Moreover, by (5.1)(5.2), we have $u_{10} / v_{10}=\left(u_{10}+u_{20}\right) /\left(v_{10}+v_{20}\right)$. Then, it follows that $u_{10} / v_{10}=u_{\text {acoustic }} / v_{\text {acoustic }}$. Thus, $u_{10} / v_{10}$ is real for solutions of the Euler equations.

\section{APPENDIX B : PML EQUATIONS FOR A MEAN FLOW IN GENERAL DIRECTION}

For a mean flow not parallel to the $x$-axis, the linearized Euler equations are

$$
\begin{aligned}
& \frac{\partial u}{\partial t}+M_{x} \frac{\partial u}{\partial x}+M_{y} \frac{\partial u}{\partial y}=-\frac{\partial p}{\partial x} \\
& \frac{\partial v}{\partial t}+M_{x} \frac{\partial v}{\partial x}+M_{y} \frac{\partial v}{\partial y}=-\frac{\partial p}{\partial y} \\
& \frac{\partial p}{\partial t}+M_{x} \frac{\partial p}{\partial x}+M_{y} \frac{\partial p}{\partial y}=-\left(\frac{\partial u}{\partial x}+\frac{\partial v}{\partial y}\right) \\
& \frac{\partial \rho}{\partial t}+M_{x} \frac{\partial \rho}{\partial x}+M_{y} \frac{\partial \rho}{\partial y}=-\left(\frac{\partial u}{\partial x}+\frac{\partial v}{\partial y}\right)
\end{aligned}
$$

where $M_{x}$ and $M_{y}$ are the mean velocities in the $x$ and $y$ directions respectively.

It has been found necessary to split $u$ and $v$ into 3 sub-components. We propose the following PML equations :

$$
\begin{aligned}
& \frac{\partial u_{1}}{\partial t}+\sigma_{x} u_{1}=-\frac{\partial\left(p_{1}+p_{2}\right)}{\partial x} \\
& \frac{\partial u_{2}}{\partial t}+\sigma_{x} u_{2}=-M_{x} \frac{\partial\left(u_{1}+u_{2}+u_{3}\right)}{\partial x} \\
& \frac{\partial u_{3}}{\partial t}+\sigma_{y} u_{3}=-M_{y} \frac{\partial\left(u_{1}+u_{2}+u_{3}\right)}{\partial y} \\
& \frac{\partial v_{1}}{\partial t}+\sigma_{y} v_{1}=-\frac{\partial\left(p_{1}+p_{2}\right)}{\partial y} \\
& \frac{\partial v_{2}}{\partial t}+\sigma_{x} v_{2}=-M_{x} \frac{\partial\left(v_{1}+v_{2}+v_{3}\right)}{\partial x}
\end{aligned}
$$




$$
\begin{aligned}
& \frac{\partial v_{3}}{\partial t}+\sigma_{y} v_{3}=-M_{y} \frac{\partial\left(v_{1}+v_{2}+v_{3}\right)}{\partial y} \\
& \frac{\partial p_{1}}{\partial t}+\sigma_{x} p_{1}=-\frac{\partial\left(u_{1}+u_{2}+u_{3}\right)}{\partial x}-M_{x} \frac{\partial\left(p_{1}+p_{2}\right)}{\partial x} \\
& \frac{\partial p_{2}}{\partial t}+\sigma_{y} p_{2}=-\frac{\partial\left(v_{1}+v_{2}+v_{3}\right)}{\partial y}-M_{y} \frac{\partial\left(p_{1}+p_{2}\right)}{\partial y} \\
& \frac{\partial \rho_{1}}{\partial t}+\sigma_{x} \rho_{1}=-\frac{\partial\left(u_{1}+u_{2}+u_{3}\right)}{\partial x}-M_{x} \frac{\partial\left(\rho_{1}+\rho_{2}\right)}{\partial x} \\
& \frac{\partial \rho_{2}}{\partial t}+\sigma_{y} \rho_{2}=-\frac{\partial\left(v_{1}+v_{2}+v_{3}\right)}{\partial y}-M_{y} \frac{\partial\left(\rho_{1}+\rho_{2}\right)}{\partial y}
\end{aligned}
$$

The solutions of (B2.1)-(B2.10) can be found analogously following section 3. In particular, the wavenumbers $k_{x}$ and $k_{y}$ of the acoustic solution are found to be

$$
k_{x}=\frac{\omega+i \sigma_{x}}{1+M_{x} \cos \phi+M_{y} \sin \phi} \cos \phi, k_{y}=\frac{\omega+i \sigma_{y}}{1+M_{x} \cos \phi+M_{y} \sin \phi} \sin \phi
$$

It can again be established that reflection is null at interfaces.

We also note that when there is no mean flow, i.e., $M_{x}=M_{y}=0$, it is not necessary to split $u$ and $v$. This will result in six equations for the PML domain by deleting (B2.2), (B2.3), (B2.5) and (B2.6).

\section{ACKNOWLEDGMENTS}

The author wishes to thank Dr. M. Y. Hussaini for his encouragements and helpful discussions. The author also wishes to thank Dr. D. G. Lasseigne for helpful comments.

\section{REFERENCES}

1. K. W. Thompson, "Time-dependent boundary conditions for hyperbolic systems", Journal of Computational Physics, 68, 1-24, 1987.

2. Giles, M. B. "Non-reflecting boundary conditions for Euler equation calculations", AIAA J., 28, 2050-2058, 1990.

3. A. Bayliss and Eli Turkel, "Radiation boundary conditions for wave-like equations", Communications on Pure and Applied Mathematics, 33, 708-725, 1980.

4. B. Enquist and A. Majda, "Radiation boundary conditions for acoustic and elastic wave calculations", Communications on pure and applied mathematics, 32 (3), 1979.

5. T. Hagstrom and S. I. Hariharan, "Accurate boundary conditions for exterior problems in gas dynamics", Math. Computation, 51, 581-597, 1988.

6. C. K. W. Tam and J. C. Webb, "Dispersion-Relation-Preserving schemes for computational acoustics", Journal of Computational Physics, 107, 262-281, 1993. 
7. D. Givoli, "Non-reflecting boundary conditions", Journal of Computational Physics, 94, 1-29, 1991.

8. C. L. Streett and M. G. Macaraeg, "Spectral multi-domain for large-scale fluid dynamics simulations", Int. Journal Appl. Numer. Math., 6, 123-139, 1989.

9. S. Ta'asan and D. M. Nark, "An absorbing buffer zone technique for acoustic wave propagation", AIAA paper 95-0164, 1995.

10. J-P Berenger, "A perfectly matched layer for the absorption of electromagnetic waves", Journal of computational Physics, 114, 185-200, 1994.

11. D. S. Katz, E. T. Thiele and A. Taflove, "Validation and extension to three dimension of the Berenger PML absorbing boundary condition for FD-TD meshes", IEEE Microwave and guided wave letters, bf 4, (8), 1994.

12. P. M. Morse and K. U. Ingard, Theoretical Acoustics, McGraw-Hill, 1968.

13. F. Q. Hu, M. Y. Hussaini and J. L. Manthey, "Low-dissipation and-dispersion Runge-Kutta schemes for computational acoustics", to appear in Journal of Computational Physics. Also ICASE Report 94-102, 1994.

14. J. C. Hardin, J. R. Ristorcelli, C. K. W. Tam (editors), ICASE/LaRC workshop on benchmark problems in computational aeroacoustics, NASA CP 3300, 1995.

15. J. Gary, "On boundary conditions for hyperbolic difference schemes", Journal of Computational Physics, 26, 339-351, 1978. 


\begin{tabular}{|l|c|}
\hline & PML \\
\hline & Mean Flow \\
\hline & interior domain \\
\hline & PML \\
\hline
\end{tabular}

Figure 1. A schematic of computational domain showing the interior domain and PML domains on the boundary.

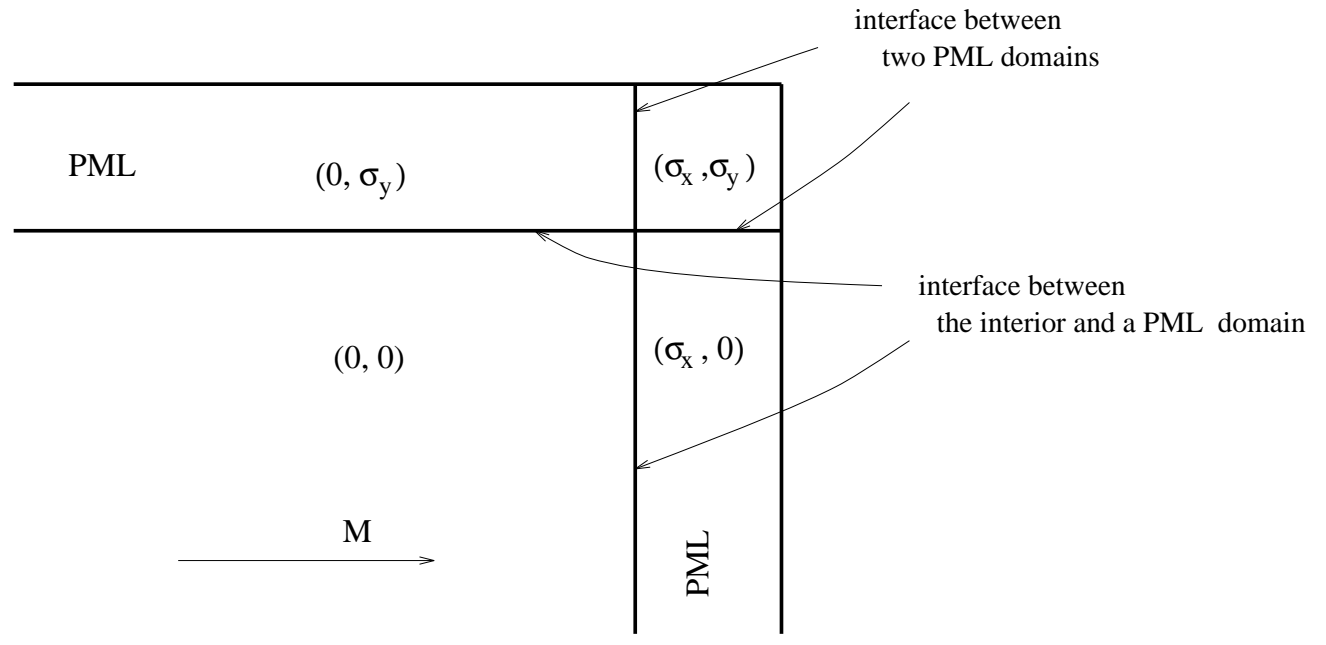

Figure 2. Absorption coefficients on a corner of the computational domain. 


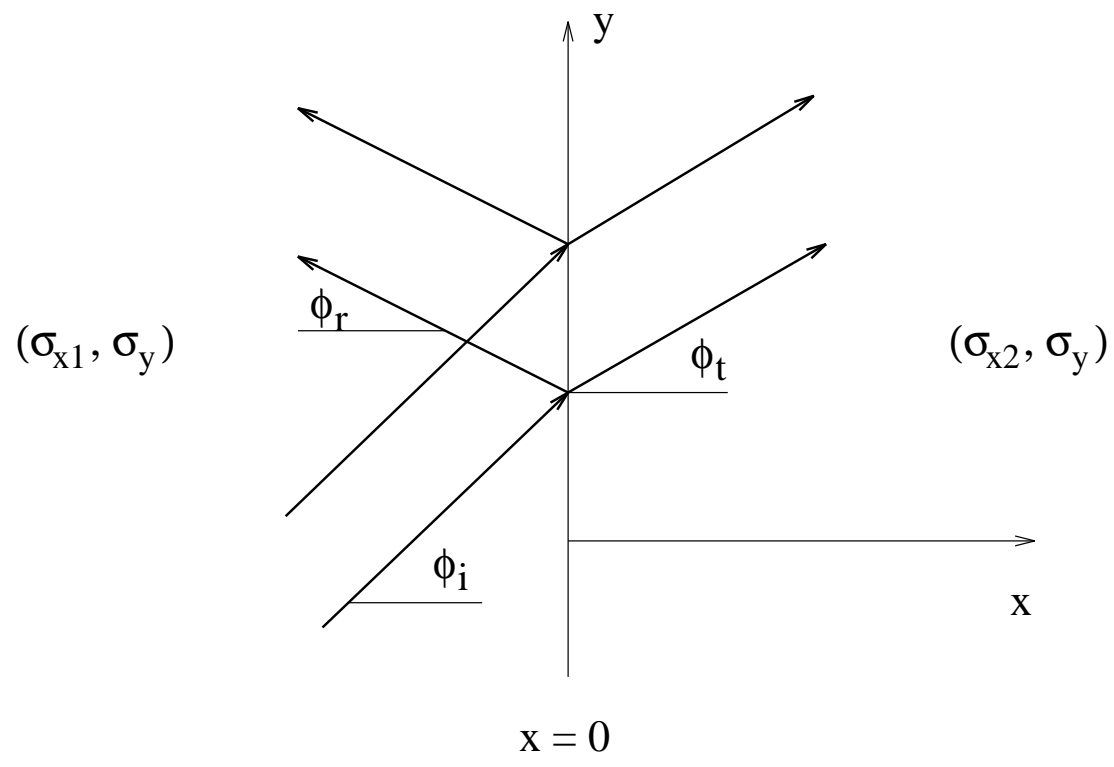

Figure 3. A schematic showing the angles of the acoustic waves at an interface. 

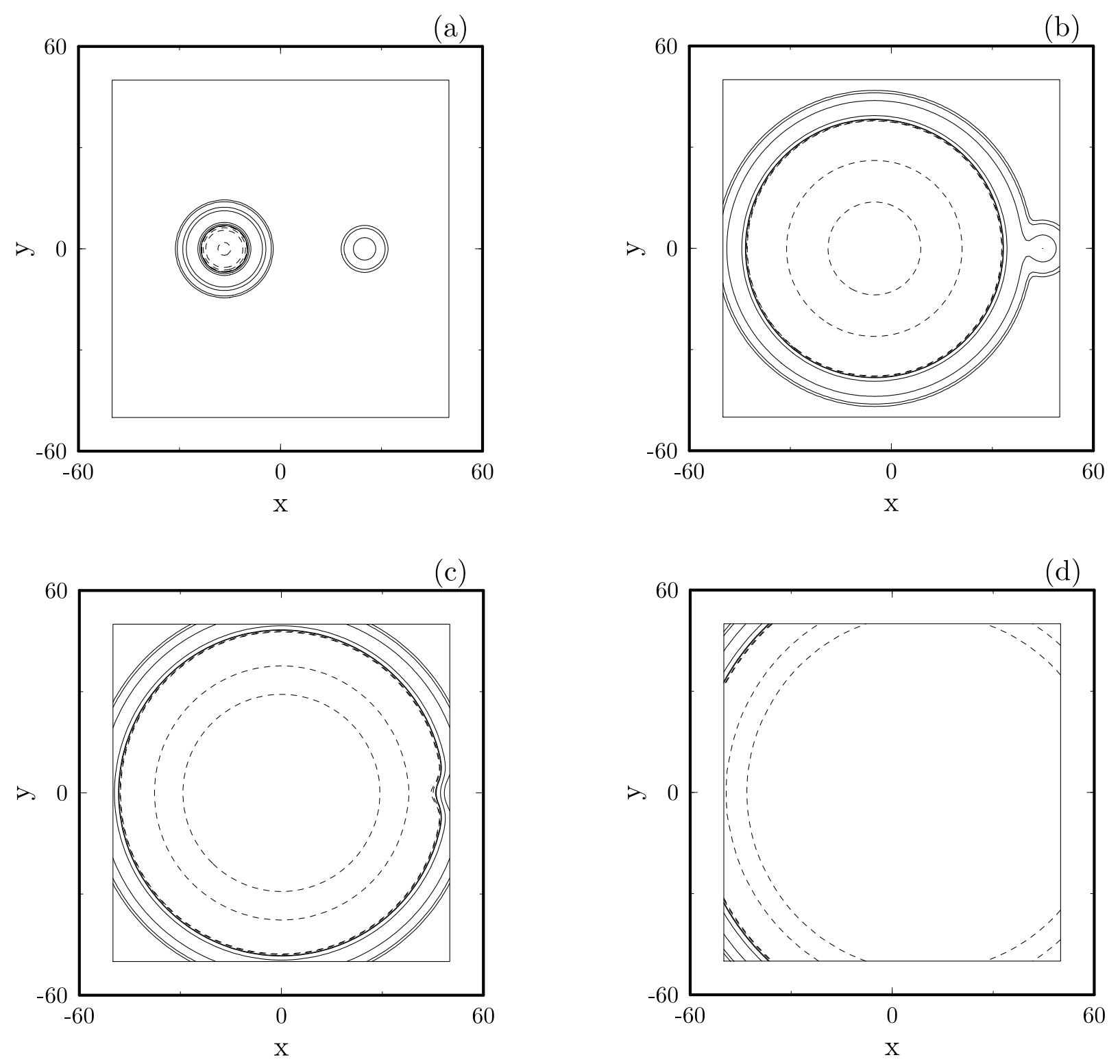

Figure 4. Density contours at values of $\pm 0.1, \pm 0.05, \pm 0.01$ and \pm 0.005 , showing the acoustic and the entropy pulses. $M=0.5$. (a) $t=10$, (b) $t=40$, (c) $t=50$, (d) $t=70$. 

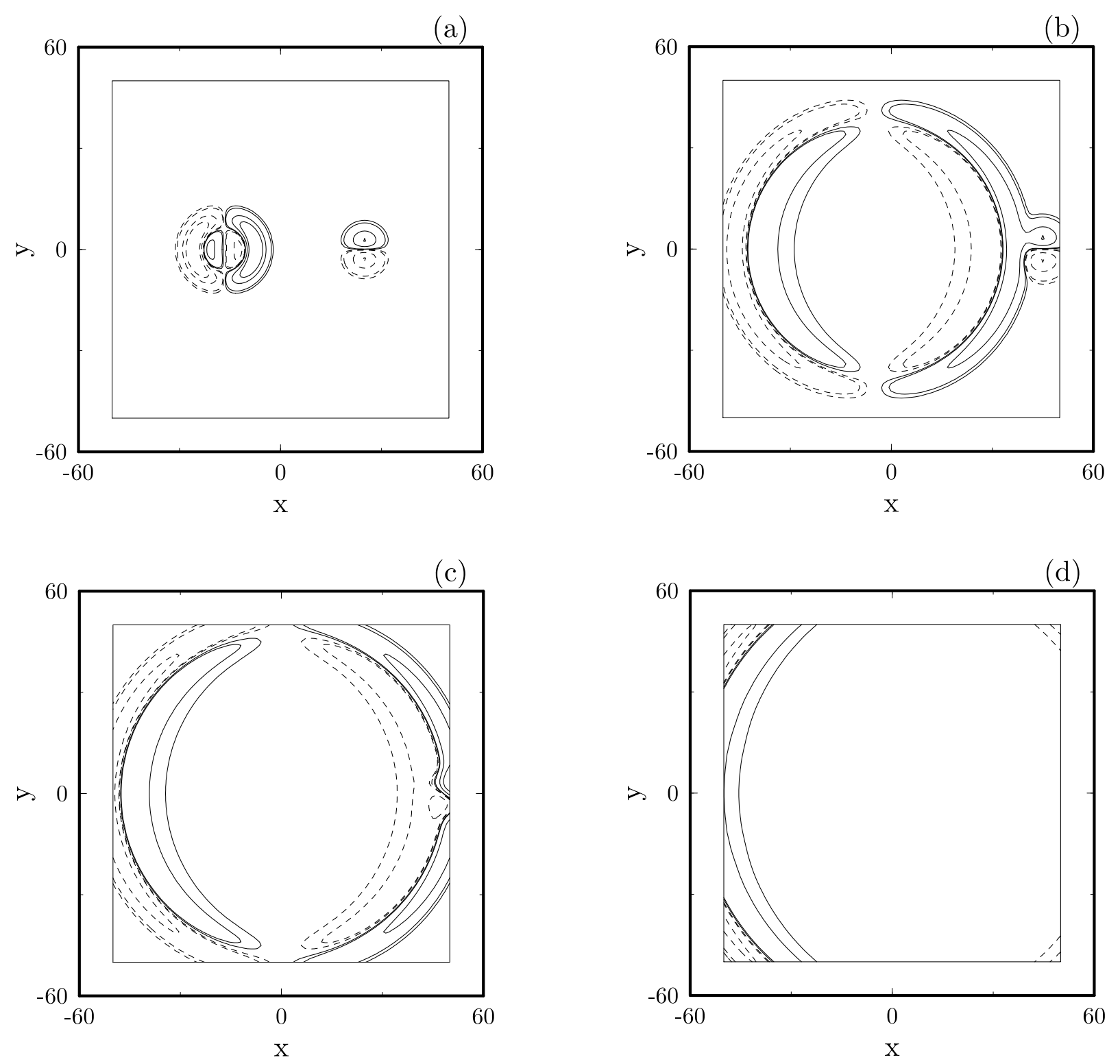

Figure 5. $u$ velocity contours at values of $\pm 0.1, \pm 0.05, \pm 0.01$ and \pm 0.005 , showing the acoustic and the vorticity pulses. $M=0.5$. (a) $t=10$, (b) $t=40$, (c) $t=50$, (d) $t=70$. 

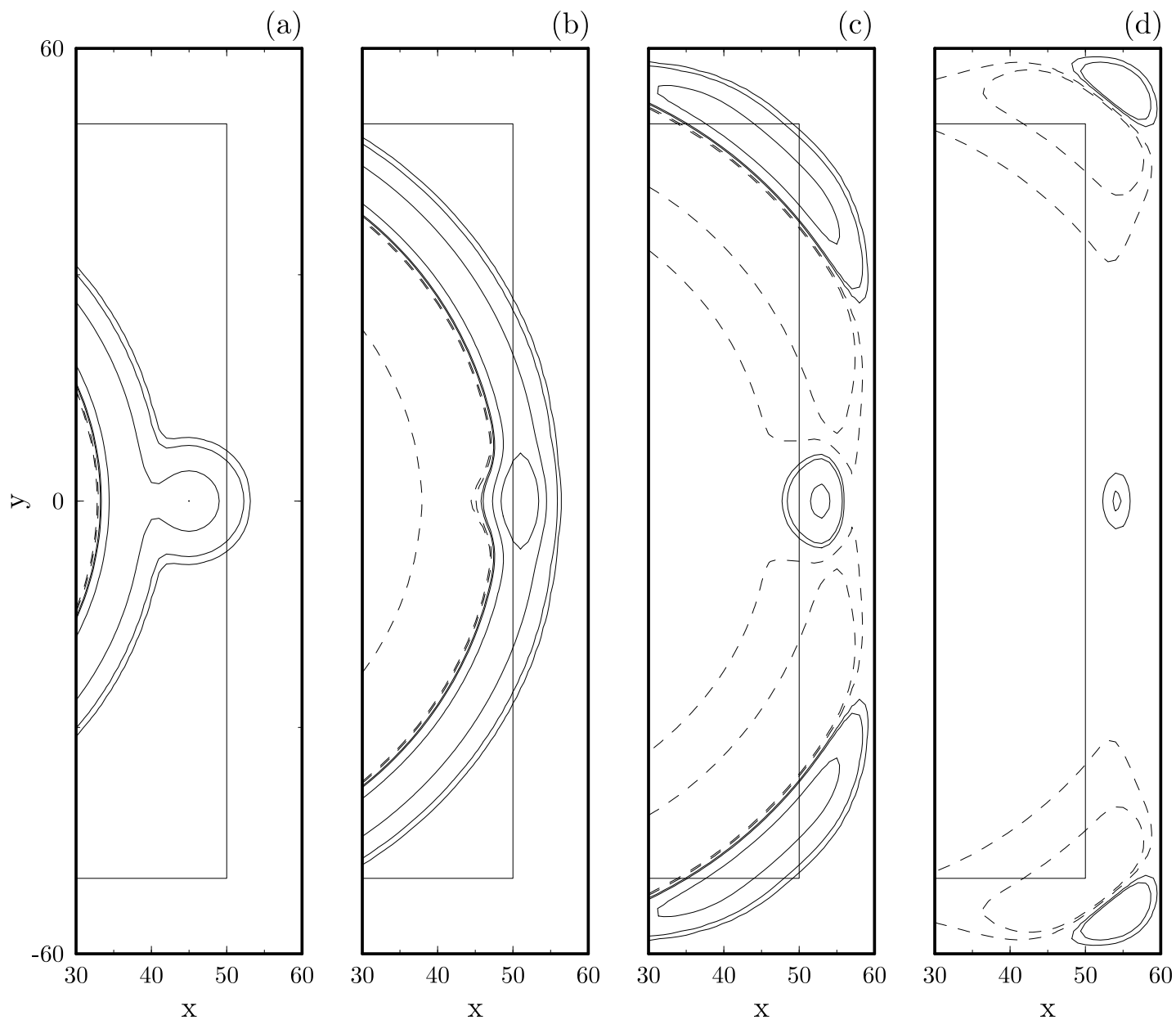

Figure 6. Density contours near the right boundary, showing the decaying of the waves in the PML domain. (a) $t=40$, (b) $t=50$, (c) $t=60$, (d) $t=70$. 

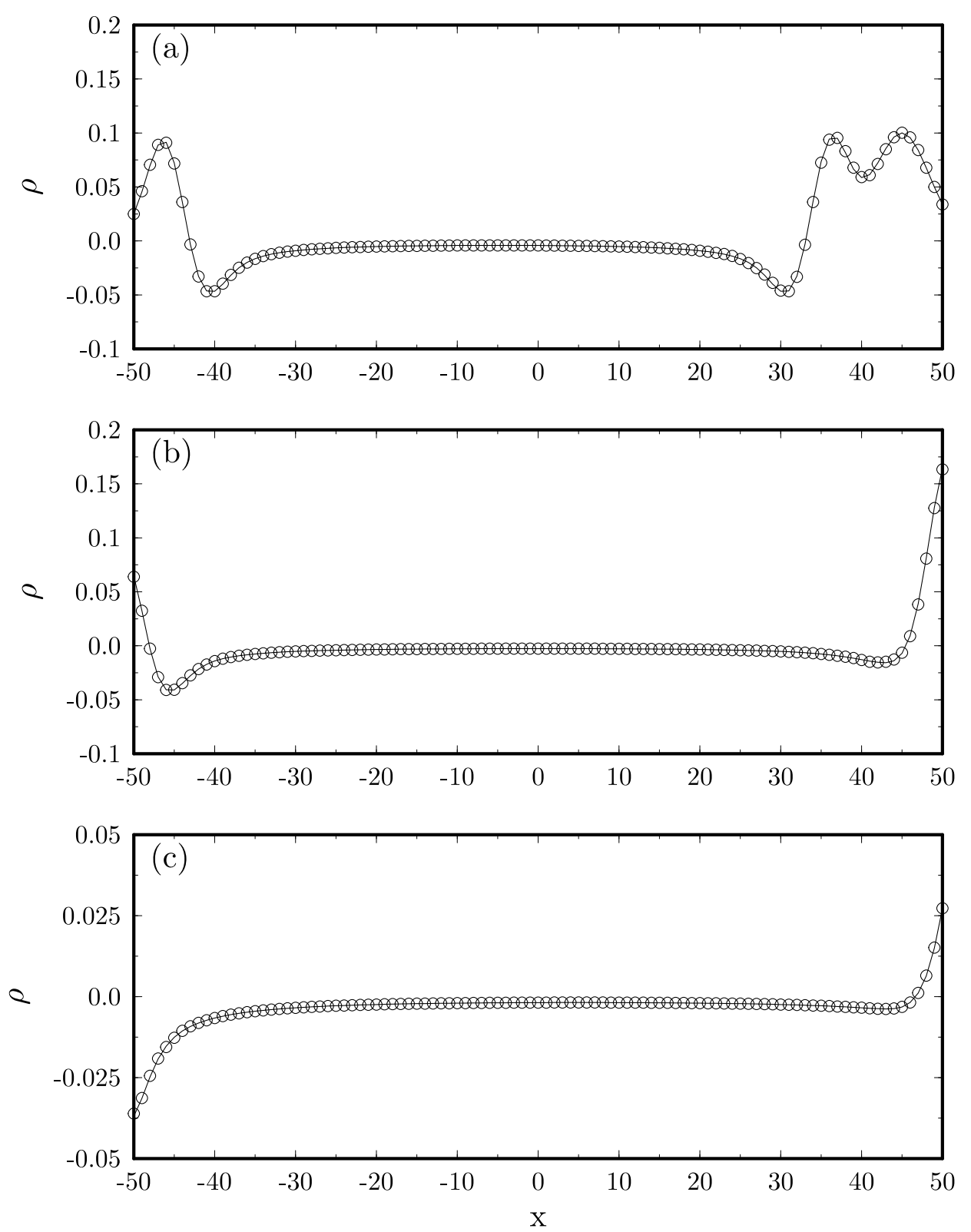

Figure 7. Density along the line $y=0 . \mathrm{M}=0.5$. exact, o numerical. (a) $t=40$, (b) $t=50$, (c) $t=70$. 

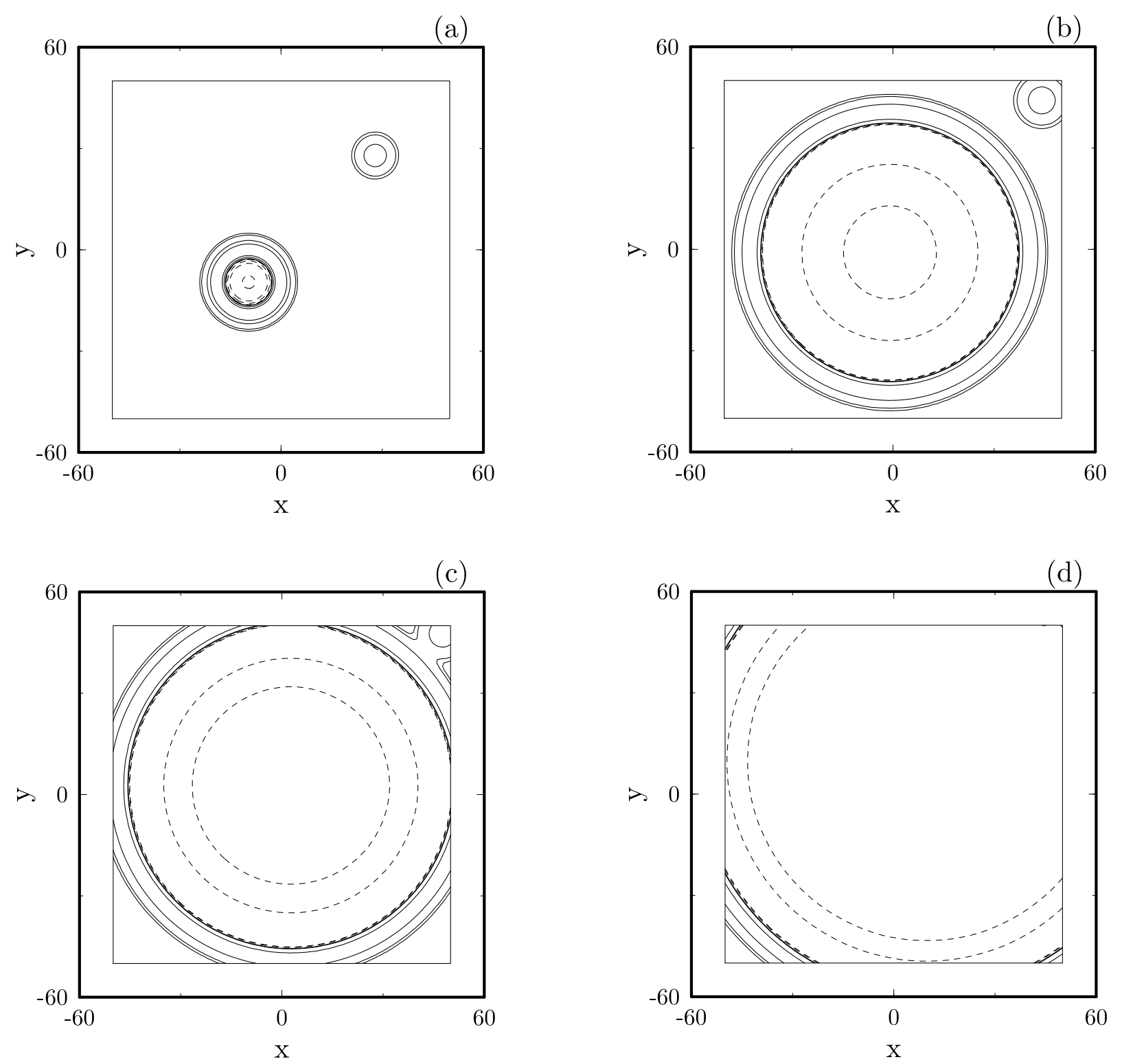

Figure 8. Density contours at values of $\pm 0.1, \pm 0.05, \pm 0.01$ and \pm 0.005 , showing the acoustic and the entropy pulses. $M_{x}=M_{y}=0.5 \sin (\pi / 4)$. (a) $t=10$, (b) $t=40$, (c) $t=50$, (d) $t=70$. 

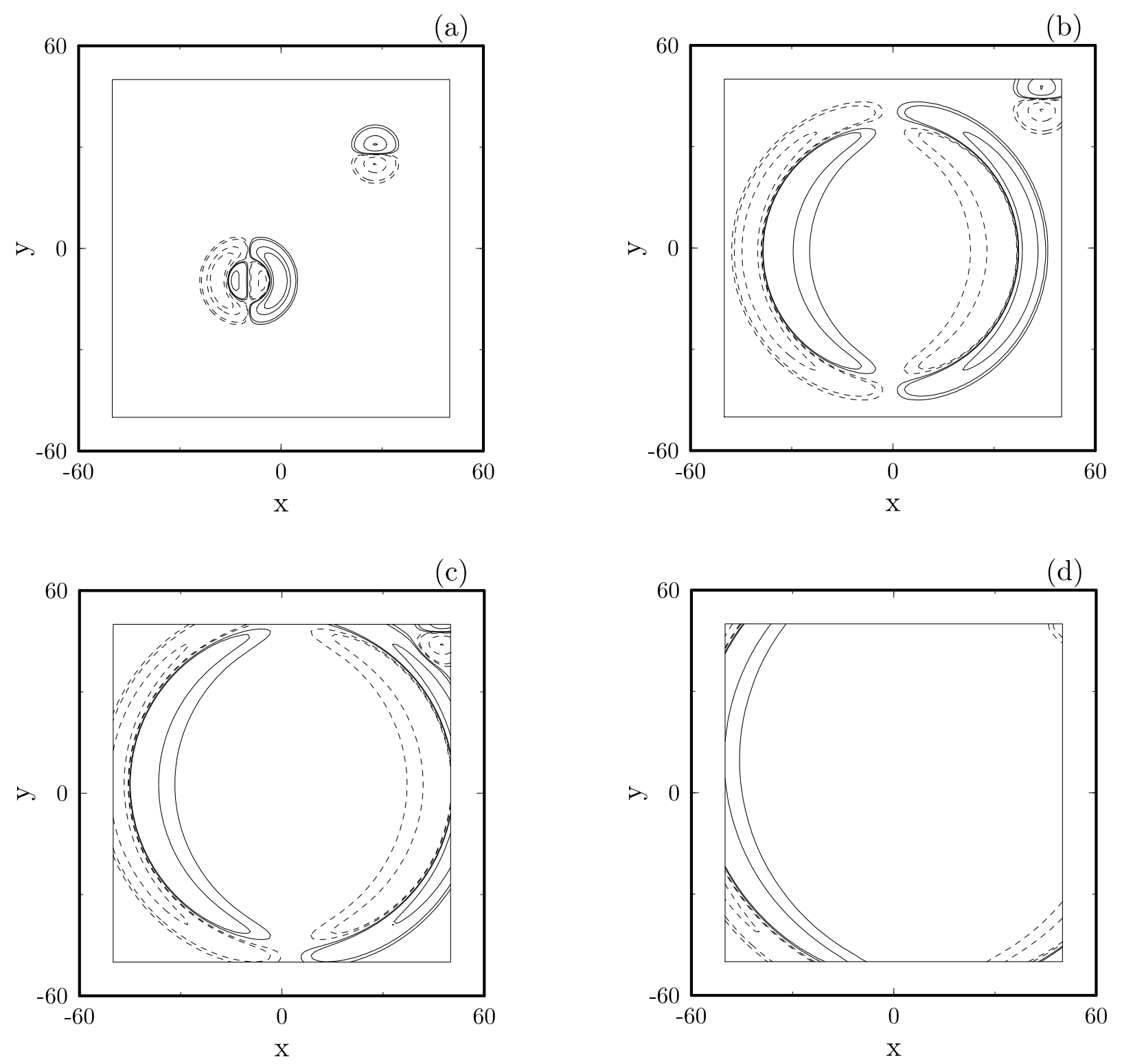

Figure 9. $u$ velocity contours at values of $\pm 0.1, \pm 0.05, \pm 0.01$ and \pm 0.005 , showing the acoustic and the vorticity pulses. $M_{x}=M_{y}=0.5 \sin (\pi / 4)$. (a) $t=10$, (b) $t=40$, (c) $t=50$, (d) $t=70$. 

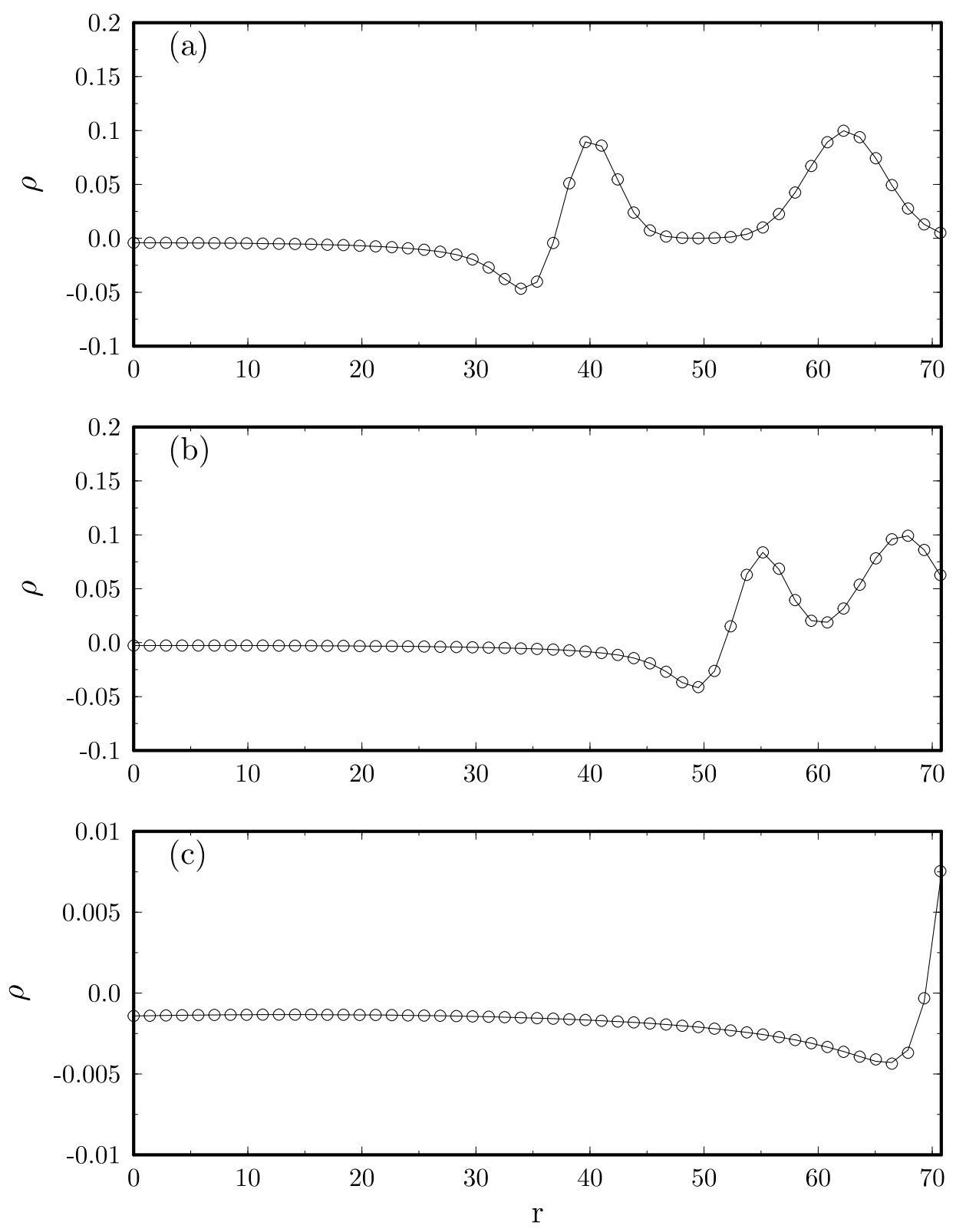

Figure 10. Density along the line $x=y . M_{x}=M_{y}=0.5 \sin (\pi / 4)$. The horizontal axis $r$ is the distance from center $(0,0)$. - exact, o numerical. (a) $t=40$, (b) $t=50$, (c) $t=70$. 


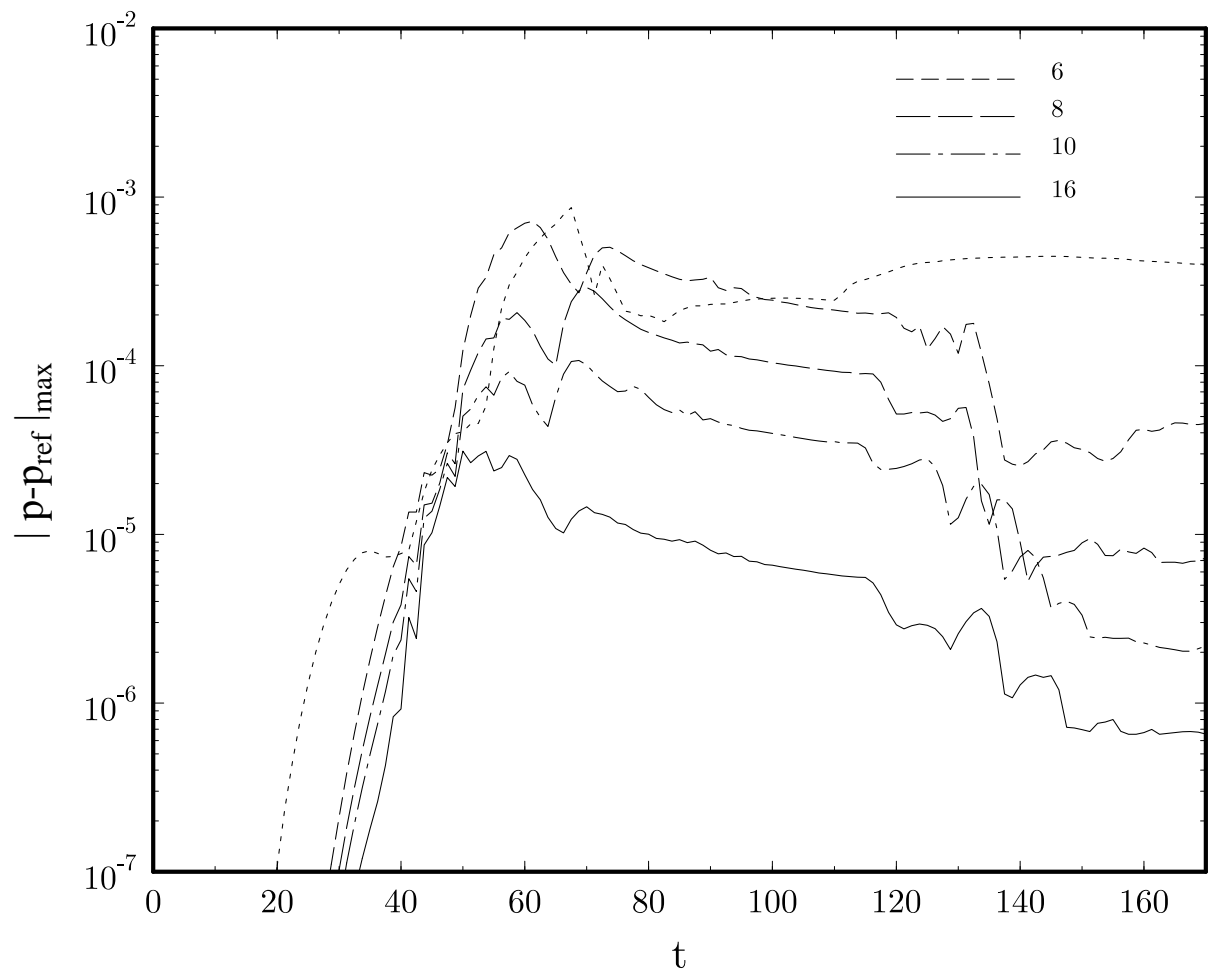

Figure 11. Maximum pressure difference of the computed and the reference solutions along $x=48$ as a function of time. $M=0.5$. Indicated are the number of grid points in PML domains used. Dotted line is the result when an asymptotic solution based boundary condition is used for $|x|$, $|y|>50$. 

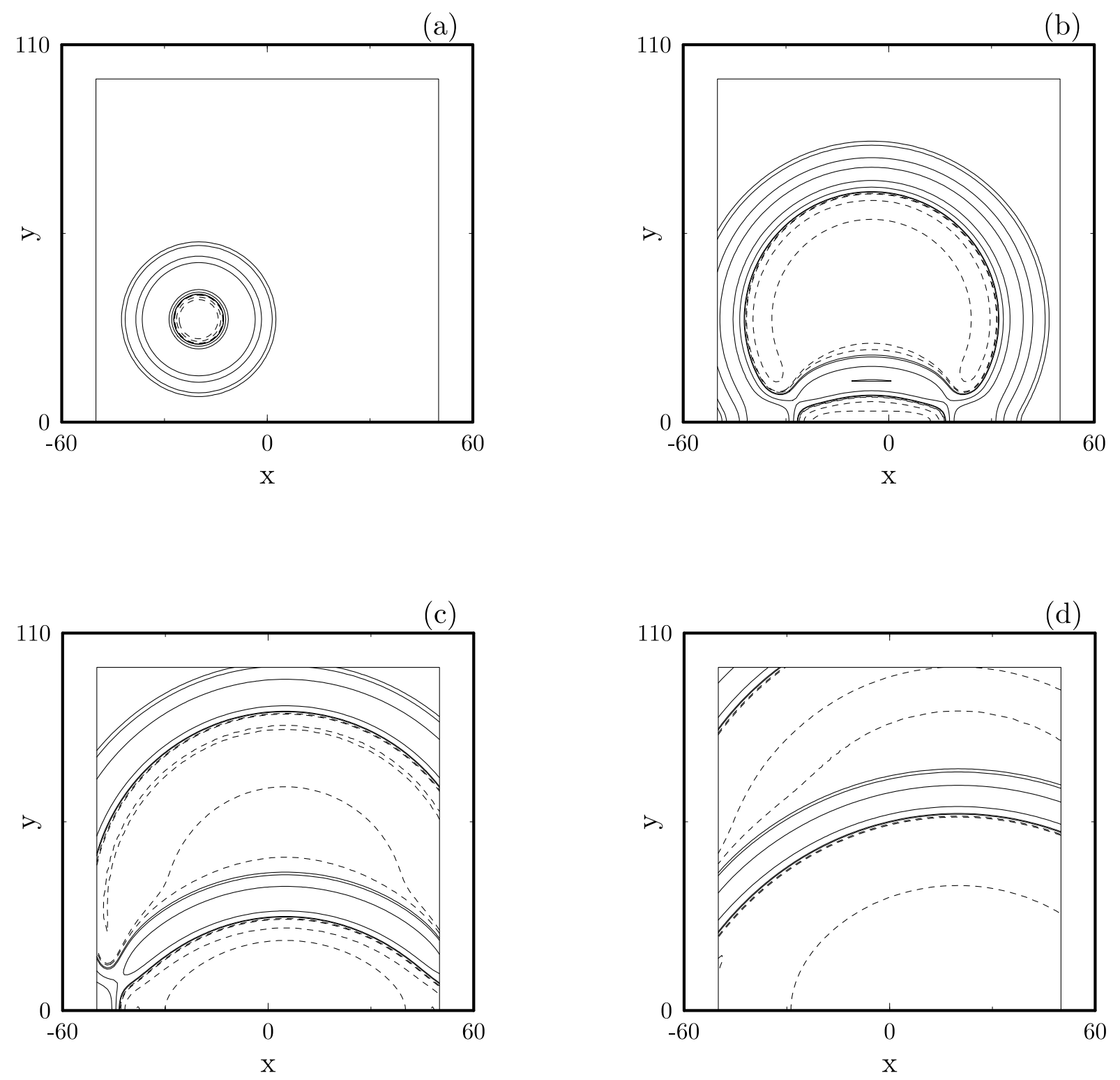

Figure 12. Pressure contours at values of $\pm 0.1, \pm 0.05, \pm 0.01$ and \pm 0.005 , showing the reflection of the acoustic pulse by a solid wall located at $y=0$. $M=0.5$. (a) $t=10$, (b) $t=40$, (c) $t=60$, (d) $t=90$. 


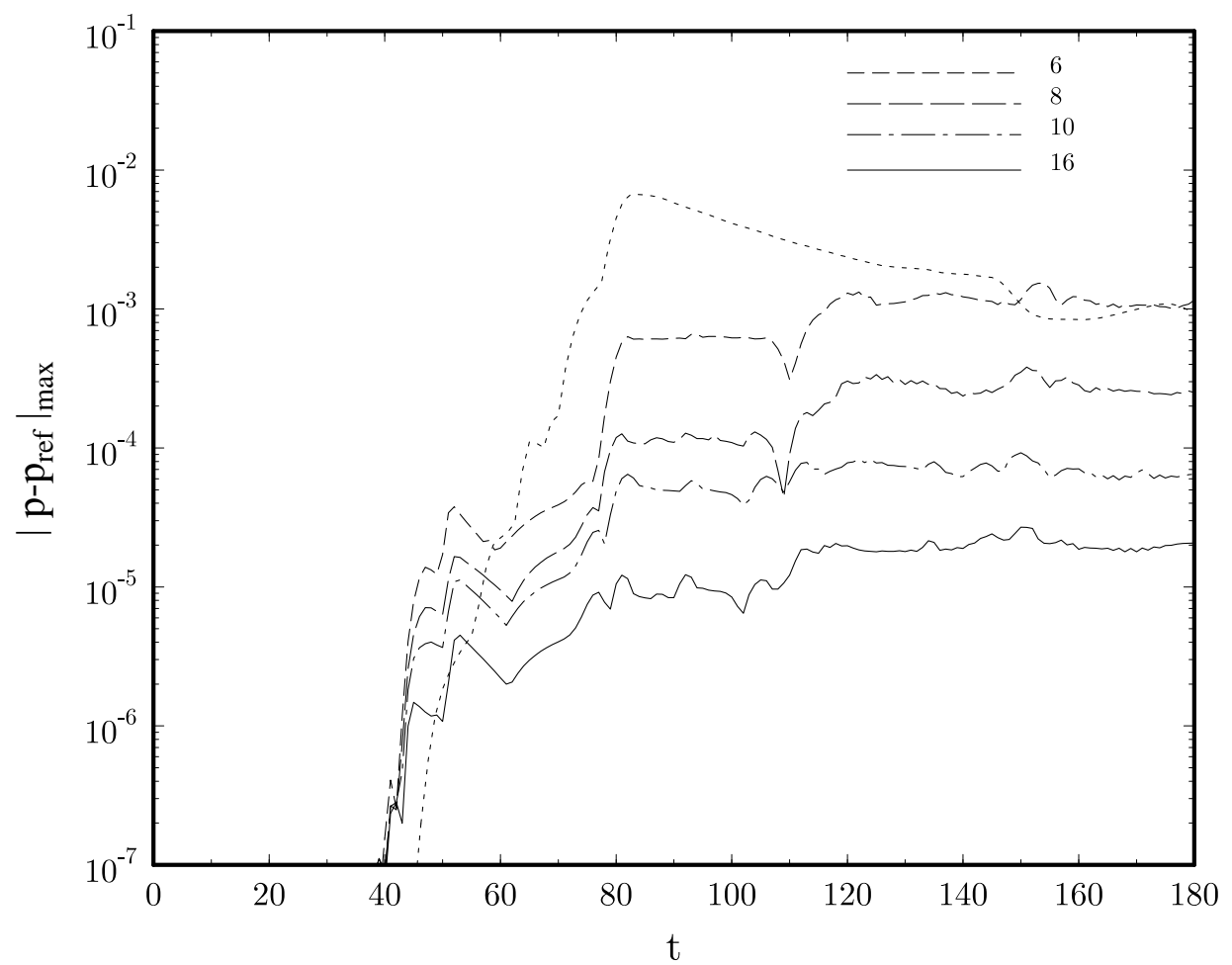

Figure 13. Maximum pressure difference of the computed and the reference solutions along $x=48$ as a function of time. $M=0.5$. A solid wall is located at $y=0$. Indicated are the number of grid points in PML domains used. Dotted line is the result when an asymptotic solution based boundary condition is used. 